\title{
Predicting subsurface sonar observations with satellite-derived ocean surface data in the California Current Ecosystem
}

Kellie R. Gadeken ${ }^{1}$, Maxwell B. Joseph ${ }^{2}$, Joseph McGlinchy² ${ }^{2}$, Kristopher B. Karnauskas $^{3,4}$, Carrie C. Wall ${ }^{4,5}$

1 Bredesen Center for Interdisciplinary Research and Graduate Education, University of Tennessee, Knoxville, Tennessee, USA

2 Earth Lab, University of Colorado Boulder, Boulder, Colorado, USA

3 Atmospheric and Oceanic Sciences, University of Colorado Boulder, Boulder, Colorado. USA

4 Cooperative Institute for Research in Environmental Sciences, University of Colorado Boulder, Boulder, Colorado. USA

5 National Centers for Environmental Information, National Oceanographic and Atmospheric Administration, Boulder, Colorado. USA

*maxwell.b.joseph@colorado.edu

\begin{abstract}
Vessel-based sonar systems that focus on the water column provide valuable information on the distribution of underwater marine organisms, but such data are expensive to collect and limited in their spatiotemporal coverage. Satellite data, however, are widely available across large regions and provide information on surface ocean conditions. If satellite data can be linked to subsurface sonar measurements, it may be possible to predict marine life over broader spatial regions with higher frequency using satellite observations. Here, we use random forest models to evaluate the potential for predicting a sonar-derived proxy for subsurface biomass as a function of satellite imagery in the California Current Ecosystem. We find that satellite data may be useful for prediction under some circumstances, but across a range of sonar frequencies and depths, overall predictive performance was low. Performance in spatial interpolation tasks exceeded performance in spatial and temporal extrapolation, suggesting that this approach is not yet reliable for forecasting or spatial extrapolation. We conclude with some potential limitations and extensions of this work.
\end{abstract}

\section{Introduction}

Sonars that focus on the water column, the volume of ocean from the near surface to near the seabed, are used widely in fisheries science and management. The backscatter (or acoustic reflectance) from these sonars help characterize the distribution of marine life beneath the surface, from zooplankton to large predatory fish, by estimating biomass [1, trophic- and species-level identification [2,3], and measuring school size and behavior 4 6]. This information can be used to understand ecosystem dynamics and inform stock assessments. However, vessel-based sonar data are expensive to collect, and have limited spatiotemporal coverage due to the constraints of the time and location of research cruises. The depth to which a water-column sonar can survey 
depends largely on the transducer frequency and pulse length. In general, lower frequency transducers (like $18 \mathrm{kHz}$ ) can record to depths exceeding $1000 \mathrm{~m}$ while higher frequency transducers (like $200 \mathrm{kHz}$ ) can only record to about $150 \mathrm{~m}$ depth 7 . Conversely, satellite-derived oceanographic data products provide information on surface (or to one optical depth) ocean conditions multiple times per day at a horizontal resolution down to $250 \mathrm{~m}$. These data potentially provide insight into surface manifestations of complex physical and biological processes.

Euphotic zone depth $\left(z_{1 \%}\right)$ is a measure of the depth where only $1 \%$ of the surface photosynthetic available radiation remains in the water column. In Case- 1 waters (which have inherent optical properties adequately described by phytoplankton, and in turn, chlorophyll concentration) remotely sensed measurements of $\mathrm{z}_{1 \%}$ from optical satellite imaging systems show good agreement with in situ measurements across the visible wavelength region for depth ranges of approximately 4-80 m 8. More conservatively, $\mathrm{z}_{10 \%}$ showed similarly good agreement in the 20-30 m depth range. Further, chlorophyll-a concentrations estimated by remote sensing reflectances show good agreement with in situ measurements, particularly for waters with $\mathrm{z}_{1} \%$ deeper than 30 meters 8 .

The California Current Ecosystem (CCE) is a coastal upwelling biome in the eastern North Pacific Ocean. This highly productive and dynamic environment supports planktonic crustaceans (euphausiids), a key food for large marine predators and a variety of commercially and environmentally critical fisheries. Aggregations of euphausiid species in the CCE, namely North Pacific krill (Euphausia pacifica), are typically found less than $30 \mathrm{~m}$ depth during the night and between 50-100 m depth during the day on the shelf and slope, and deeper offshore 9 . This diel vertical migration (daily movement to disperse in shallower waters at night and aggregate at depth during the day) is a well-known, predictable behavior driven by feeding and predator avoidance behavior. These patterns can also be easily discerned from water-column sonar data to effectively identify and map their distribution [7,9 91$]$.

Coastal pelagic fish species in the CCE include jack mackerel (Trachurus symmetricus), Pacific mackeral (Scomber japonicus), and Pacific sardine (Sardinops sagax), which are most commonly found between 0-50m depth but can occur down to $300 \mathrm{~m}$ and $100 \mathrm{~m}$, respectively [12. Northern anchovy (Engraulis mordax) and Pacific herring (Clupea pallasii) are distributed from 0-200 $\mathrm{m}$ depth 12 . Pacific hake (Merluccius productus) are found down to $600 \mathrm{~m}$ depth but most commonly between 200-500 m 12 13. Pacific saury (Colalabis saira) is also found in the CCE down to 250 $\mathrm{m}$, though away from the coast and limited to Central and Northern California [12]. With the exception of Pacific hake, these species undergo diel vertical migration 12 .

If satellite data can predict subsurface biomass measured by sonar, it may be possible to predict marine life over broader spatial regions with higher frequency using satellite observations. The first step toward such prediction is to evaluate relationships between satellite observations and acoustic returns. This approach has been demonstrated for Pacific sardine (Sardinops sagax) in the CCE [14]. A key challenge is to identify methodology to analyze these temporally and spatially diverse datasets for general biomass (non-species specific) distributions.

Here, we adopt a data-driven approach to explore whether satellite measurements of ocean surface characteristics can predict observations from water-column sonar data collected in the CCE over a 5 year period, which includes the 2015/16 El Niño. We use random forests to learn a functional mapping from satellite and physical explanatory variables to sonar data previously collected as part of National Oceanic and Atmospheric Administration (NOAA) National Marine Fisheries Service (Fisheries) and Fisheries and Oceans Canada (DFO) efforts to quantify Pacific Hake (Merluccius productus) biomass. We evaluate the ability of these models to interpolate and extrapolate in space and time 


\section{Materials and methods}

\section{Sonar data}

Water-column sonar data were collected on NOAA Ship Bell M. Shimada by NOAA Northwest Fisheries Science Center (NWFSC) scientists to inform management decisions for Pacific Hake fisheries. NWFSC-DFO surveys ("Joint U.S.-Canada Integrated Acoustic Survey of Pacific Hake") are focused in the boreal summer (largely July-August) along the Pacific coast of the United States and Canada. These surveys use systematic transects that result in approximately overlapping cruise tracks between surveys at approximately the same time of year. A Simrad EK60 split-beam echosounder with $18 \mathrm{kHz}, 38 \mathrm{kHz}, 70 \mathrm{kHz}, 120 \mathrm{kHz}$, and $200 \mathrm{kHz}$ transducers was used on NWFSC cruises conducted in 2011, 2012, 2013 and 2015 15 18. These data were archived at the NOAA National Centers for Environmental Information where they are publicly accessible and subsequently used in this analysis. The NWFSC 2014 cruise was not included in the analysis due to its sparse spatial coverage compared to the other four cruises.

Prior to each cruise, the echosounder was calibrated using the standard sphere method [7, 19]. Target strength and echo integration data were collected to calculate echosounder gain parameters to ensure the quality of the system performance.

On-axis 7 beam-pattern measurements were also taken. To minimize the effect of surface bubbles and transducer "ring down", acoustic data were collected from $10 \mathrm{~m}$ below the surface, roughly $5 \mathrm{~m}$ below the centreboard-mounted transducer face. Data were collected to a maximum depth of $750 \mathrm{~m}$. With the transducer depth accounted for, the volume backscatter strength $\left(\mathrm{S}_{V}\right)$ sample depth is relative to the sea surface. This recording range resulted in a ping rate of 1 ping per $1.1 \mathrm{~s}$.

All files were processed using Echoview (Myriax, 10) by first aligning pings in the time/distance domain across the frequency components. Data were then binned vertically to 1000 data points between 0 and $750 \mathrm{~m}$ (i.e., $\mathrm{S}_{V}$ at $0.75 \mathrm{~m}$ intervals). Noise filters were applied to remove background noise and intermittent impulsive noise.

Background noise was removed following [20] where the signal-to-noise ratio was set to $10 \mathrm{~dB}$. Impulsive noise "spikes" were removed following 21] where a ping was removed if the $\mathrm{S}_{V}$ was $10 \mathrm{~dB}$ higher or lower than the adjacent pings. Pings associated with transient noise and attenuated signal were removed following [21. The transient noise algorithm identifies and adjusts sample values that are significantly higher than those of surrounding samples, namely $10 \mathrm{~dB}$ above a $5 \times 9$ sample window. The attenuated signal algorithm identifies pings with decreased signal strength $(10 \mathrm{~dB})$ relative to the 3 surrounding pings. Additional noise or data of questionable quality due to transmission loss were also removed. These parameters were based on empirical evidence by transducer frequency: $70 \mathrm{kHz}$ data collected in depths beyond $500 \mathrm{~m}$ were removed, 120 $\mathrm{kHz}$ data were removed beyond $275 \mathrm{~m}$ depth, and $200 \mathrm{kHz}$ data were removed beyond $150 \mathrm{~m}$ depth. Acoustic reflections from the seafloor were delineated using Echoview's

"best bottom candidate" automated algorithm focused on a peak threshold of -30 dB to distinguish between the water column and seafloor. Data were removed up to $5 \mathrm{~m}$ above the detected bottom to help account for imperfections in the seafloor detection.

The acoustic dataset resulted in over 33,000 files. As such, it was impractical to manually scrutinize the seafloor detection and completely remove all unwanted/non-biological acoustic signal. The above automated processes aimed to remove as much noise as possible in an efficient manner. However, the persistence of a fraction of the original noise is expected. By examining the data over a large scale, we 
anticipate the influence of any remaining noise to be insignificant compared to the overall signal.

After removing data associated with noise and the seafloor, the water-column sonar backscatter $\left(\mathrm{S}_{V}\right)$ was integrated into $4 \mathrm{~km}$ horizontal bins and vertical bins of 10-50 m depth ("shallow water"), 50-200 m ("mid water"), 200-500 m ("shelf water"), and 500-750 m ("deep water") for each frequency at a threshold of $-80 \mathrm{~dB}$. This undifferentiated integrated backscatter (acoustic energy represented by nautical area scattering coefficient $\left(\mathrm{s}_{A}, \mathrm{~m}^{2}\right.$ nautical mile $\left.{ }^{-2}\right)[22$, hereafter referred to as NASC, provides a proxy for biomass across the cruise track at depths that align with anticipated biological features (shallow water: epipelagic layer, near surface euphausiids and other zooplankton layers, small fish, and night-time distribution; mid water: end of epipelagic layer, overlap with euphausiids, and small fish, and possible hake distribution; shelf water: start of mesopelagic layer, some euphausiid sp. and typical depth for hake; and deep water: deep fish assemblages) 9, $10,13,23$. Due to the lack of classification of the backscatter, the results will not inherently distinguish between species or trophic levels. However, the examination of the variability of acoustic energy across the frequencies has been used to discern species 24,25]. Size and certain characteristics (swim bladder vs. non-swimbladder) of marine organisms influence its frequency response curve (how strongly sound reflects at discrete frequencies). In general, large swim-bladdered fish reflect strongest at low frequencies $(18$ and $38 \mathrm{kHz})$ while small euphausiids reflect strongest at high frequencies (120 and $200 \mathrm{kHz}) 3$. Myctophid fish, present throughout the CCE, of course, do not follow these generalizations and, while small, reflect strongly at low frequencies [26]. The horizontal extent of the NASC values align with the satellite data described below.

\section{Satellite data}

We acquired $4 \mathrm{~km}$ resolution chlorophyll-a concentration, normalized fluorescence line height, particulate organic carbon, and sea surface temperature (SST) from MODIS (Terra and Aqua) level-3 ocean color standard mapped image products. The satellite data followed the same spatial extent where the sonar data were collected and averaged using a 7 day window (+/- 3 days from the date of sonar data collection) using Google Earth Engine 27]. Observations from Terra and Aqua collected on the same day were averaged. We then averaged observations from the 7 day window to derive a mean value for each satellite variable at each $4 \mathrm{~km}$ sonar aggregation bin. Finally, we filtered the data to exclude sonar observations for which no satellite data were available. The optical column depth contributing to MODIS observations will vary with water column conditions, however, we expect the shallower depths of the sonar data to be most relevant to the satellite derived observations 8 .

\section{Other explanatory variables}

In addition to the oceanographic variables derived from MODIS satellite data, we used geographic and observation-level variables to help explain the sonar backscatter data (Table 1). Geographic variables included distance from shore, and ocean depth derived from the general bathymetric chart of the ocean (GEBCO) 201430 arc-second grid bathymetry dataset 28 . Observation variables include characteristics associated with the sonar data collection, including a binary indicator for whether the data were collected during the day or night, and categorical indicators for wavelength, depth bin, and wavelength-depth bin combinations. 
Table 1. List of explanatory variables used in the random forest models. For each variable, we list its name, type, source, and whether it was included in the reduced model. The full model includes all explanatory variables.

\begin{tabular}{llll} 
Explanatory variable & Type & Source & Reduced model \\
\hline Daytime & Binary & Sonar data & yes \\
Distance from shore & Real & Sonar data & yes \\
Frequency & Categorical & Sonar data & yes \\
NASC depth bin & Categorical & Sonar data & yes \\
Latitude & Real & Sonar data & yes \\
Ocean depth & Real & GEBCO & yes \\
Chlorophyll a & Real & MODIS & no \\
Normalized fluorescence line height & Real & MODIS & no \\
Particulate organic carbon & Real & MODIS & no \\
Sea surface temperature & Real & MODIS & no
\end{tabular}

\section{Random forests for sonar prediction}

We used random forest models to predict NASC as a function of the explanatory variables. We considered two types of models. The full model included all explanatory variables. The reduced model included only the geographic and observation covariates, and no satellite covariates. We used 100 trees each, a minimal node size of five, and unlimited tree depth. Because the distribution of NASC values is heavy-tailed, and the random forest regression models used mean-squared-error loss, all models were trained to predict NASC on a $\log _{e}$ scale. By comparing the performance of these models, we can evaluate whether satellite data are useful for predicting acoustic biomass.

\section{Model evaluation}

We used two strategies to evaluate predictive performance. First, to evaluate predictive performance in unsampled spatial regions, we used geographic 10-fold cross validation, binning by latitude using data from 2011, 2012, and 2013. Here, the data are split into 10 latitude bins, and each fold is withheld, training a model using all of the data except for the data in the withheld fold. Then, we evaluated the ability of the model to predict the data in the withheld latitude bin, repeating this process 10 times, once for each latitude bin. The results of this 10 -fold cross validation provide insight into predictive performance in unsampled spatial locations. Second, to evaluate predictive performance in unsampled years, we withheld the final year of data (2015) as a test set, and trained the model using all of the data from 2011, 2012, and 2013. We note that 2015 provides a particularly challenging out-of-sample test set, because of the presence of a marine heat wave in the Pacific 29]. The combination of these two strategies provide insight into the generalization ability of our model in space and time respectively. We evaluate performance in terms of the coefficient of determination $R^{2}$ :

$$
R^{2}(y, \hat{y})=1-\frac{\sum_{i=1}^{n}\left(y_{i}-\hat{y}_{i}\right)^{2}}{\sum_{i=1}^{n}\left(y_{i}-\bar{y}\right)^{2}}
$$

where $y_{i}$ is the $i^{\text {th }}$ observed value of $\log$ NASC, $\hat{y}_{i}$ is a predicted value, $\bar{y}$ is the sample mean of observed values, and $n$ is the number of observations. This measure of performance can be negative. For ease of visualization we set negative $R^{2}$ values to 0 in subsequent discussion, where a 0 value indicates the worst possible value. To understand how predictive performance varied as a function of sonar frequency and depth bin, we compute $R^{2}$ values for model-frequency-depth bin combinations. 
The location of sonar data, latitude bins (for the cross-validation), and test data are shown in Fig 1. All models were fit in $\mathrm{R}$ version 3.6.2 using the ranger package 30,31 . The data are publicly available via figshare [32], and a research compendium is available on GitHub at https://github.com/earthlab/ccesonar.

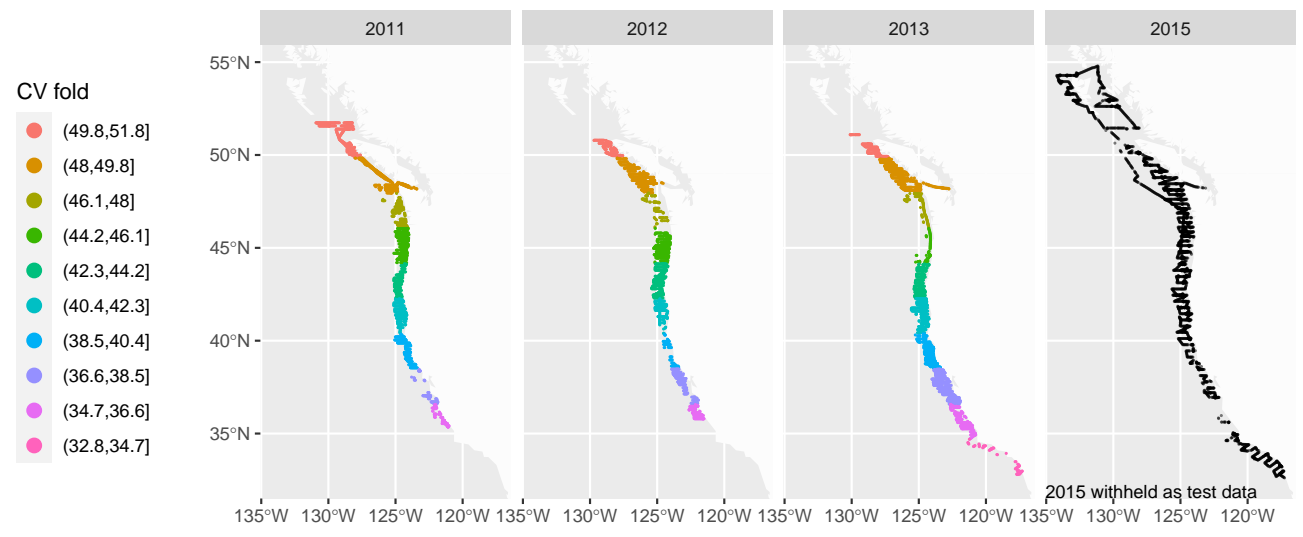

Fig 1. Acoustic data coverage by year. Color represents the ten cross validation (CV) folds that correspond to latitudinal bins. Each point represents a spatial location along a trackline where data were collected. The 2015 points are black, and represent a withheld test set that was not used in spatial cross validation.

\section{Results}

The performance of the full and reduced models indicate that satellite data may help to predict sonar observations under some circumstances, though the overall performance of all models was relatively low. Across all depth bins and frequencies, holdout $R^{2}$ values from spatial cross-validation range from 0 to 0.315 (mean $=0.059$ ) for the full model, and 0 to 0.245 (mean $=0.042$ for the reduced model). For many of the latitude, sonar frequency, depth bin combinations, performance of both models was quite poor (Fig 22 . For the shallow water depth bin, where we expected the satellite data to provide the most useful information, performance of the full model tended to be higher than performance from the reduced model for a subset of the latitude bins, and $R^{2}$ for the shallow water bin exceeded 0.1 just once south of 40.4 degrees latitude. Notable decreases in predictive performance are apparent in the extreme southernmost and northernmost latitude bins (Fig 2).

All raw $R^{2}$ values for the test set were less than zero for the full and reduced models (and were set to zero after rescaling as described in the methods). To investigate this, we computed correlations between the observed and predicted values by depth bin and sonar frequency, and visualized the relationship between observed and predicted values. In one case, the model predictions were actually negatively correlated with the true values, but all other correlations between the true and predicted values were positive (Fig 3). Notably, the full model consistently outperformed the reduced model for the shallow depth bin in terms of correlation coefficients (Fig 3). However, $R^{2}$ values were negative or zero because of systematic downward bias in model predictions. Specifically, the true average $\log$ NASC values were always higher than the predicted averages (Fig 4. 


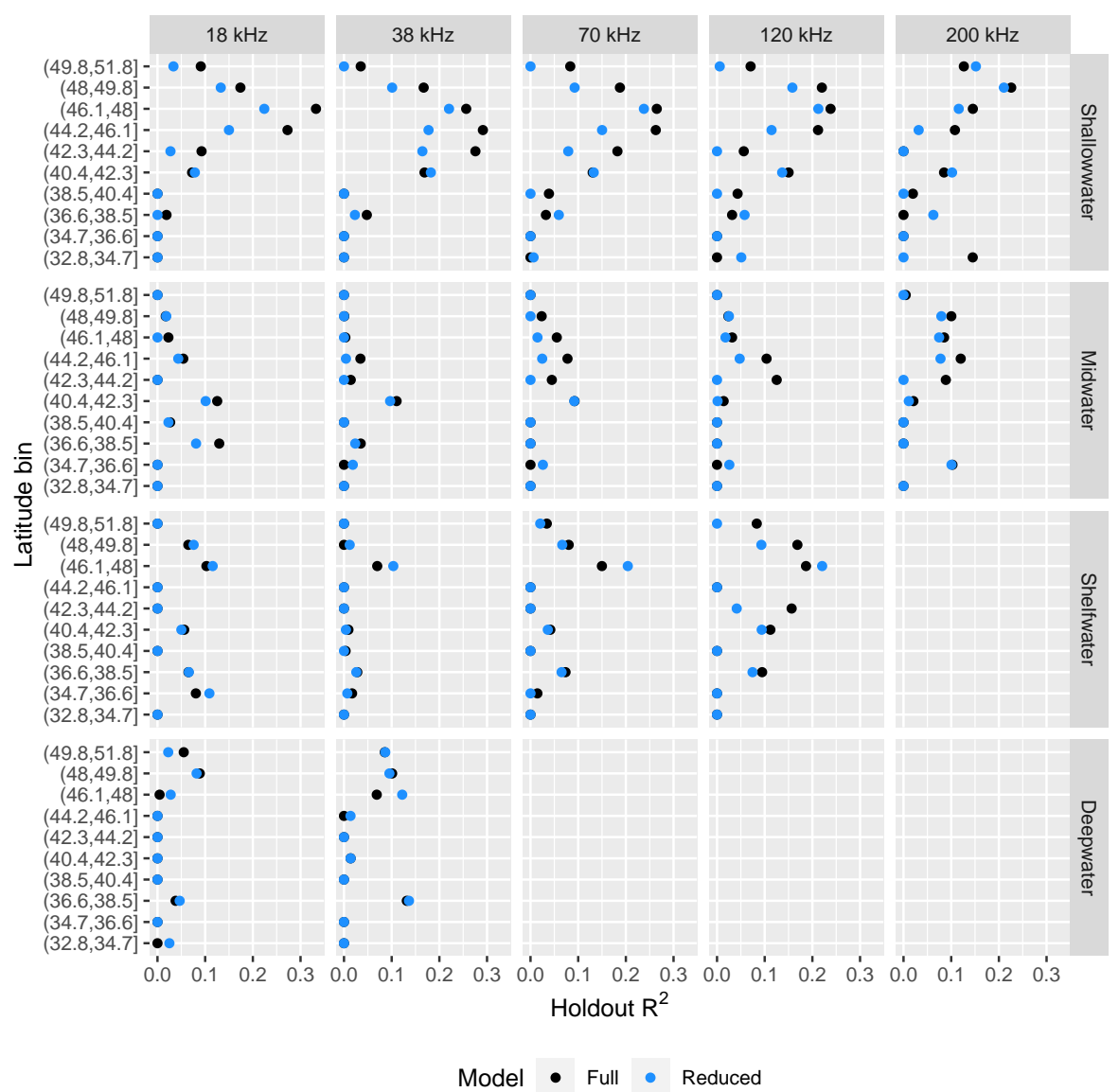

Fig 2. Spatial cross-validation. Spatial cross-validation performance comparison for the full model (black) inclusive of satellite observations, and the reduced model (blue) that excludes satellite observations, faceted by depth bin and sonar frequency $(\mathrm{kHz})$. The x-axis displays the $R^{2}$ value on the withheld latitude bin, and the y-axis displays the latitude bin that was withheld during cross-validation. No results are shown for depth bin/frequency combinations for which the sonar data are known to be unreliable.

\section{Discussion}

We found equivocal evidence that satellite data can predict water-column sonar observations. Based on this exploratory study, it seems that satellite observations are most useful when interpolating spatially, and less useful when extrapolating in space and time. This finding is supported by 1) the difference in out-of-sample predictive performance between the spatial cross-validation and two-years-ahead predictions for the 2015 test set, and 2) the decrease in spatial cross-validation performance in the extreme northern and southern latitude bins, which involve spatial extrapolation.

Overall, sonar observations were hard to predict with high accuracy. The shallowest depth bins had the highest predictive performance, which suggests that information provided by the MODIS data products is more relevant for surface observations than observations in mid to deep water. Spatiotemporal dynamics of fish and plankton are complex and non-stationary. Inclusion of sustained features, such as chlorophyll and sea surface temperature fronts, would be beneficial in future work as these conditions are known to influence biogeography and the distribution of marine organisms [33, 34]. 

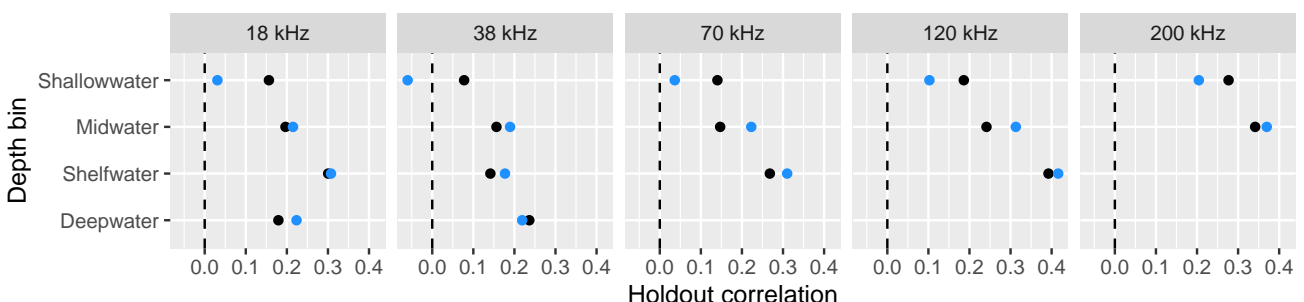

Model • Full • Reduced

Fig 3. Withheld 2015 test set correlations. Pearson correlation coefficients between the true and predicted log NASC values in the 2015 test set, with black representing the full model and blue representing the reduced model. The $\mathrm{x}$-axis represents the correlation values, and the y-axis represents depth bins. The panels represent the sonar frequency $(\mathrm{kHz})$.

Poor performance in the withheld 2015 test set might be a consequence of anomalous conditions in the CCE that resulted from a strong El Niño event that occurred from 2015 into 2016, with a particularly strong Kelvin wave and atmospheric response 35 36. Impacts on the local circulation and ecosystem within the CCE have been explained 37] and reported by in situ measurements [38]. In particular this event brought anomalies in both SST and Chlorophyll a over much of the study region [37, such that the data withheld in the 2015 test set contained SST and Chlorophyll a values near or outside the range of values in the 2011-2013 training data. As a result, the predictions for 2015 likely represent a combination of extrapolation forward in time and extrapolation in the space of the model inputs (SST, Chlorophyll a) for any particular spatial location.

Geographic misalignment provides another potential explanation for poor predictive performance. Specifically, there was potential for geographic inaccuracies in the aggregation of the sonar data to the spatial resolution of the MODIS product. The sonar data are recorded and stored as irregular point data, and when aggregated to 4 $\mathrm{km}$ to match the MODIS products there was no guarantee that the aggregation window matched exactly that of a single MODIS grid cell. Properly aligning the sonar aggregations to the MODIS grid, or that of any other satellite data used for ocean color analysis, might prove advantageous in subsequent work.

Another potential limitation for the random forest models might be the use of raw explanatory variable values, rather than deviations from long term averages. Such anomalies may be more useful but would involve a translation (typically subtracting the mean) of all relevant explanatory variables. Anomalies allow the effects of explanatory variables to vary spatially (depending on how they deviate from the mean), and random forests similarly allow the effects of explanatory variables to vary as functions of other covariates such as latitude and longitude. However, the data examined here spans only 5 years and a longer time series of input data and climatology from which to determine anomalous conditions could be informative.

Previous work has predicted in situ ocean observations as a function of satellite observations using machine learning. For example, MODIS-derived sea surface temperature and chlorophyll a have been combined with random forests to predict sea surface salinity 39. Similarly, MODIS satellite observations have also been used to identify harmful algal blooms [40. Zwolinski et al. 14 developed predictive habitat maps for spawning Pacific sardine following optimal ranges of satellite-derived sea surface temperature, chlorophyll a concentrations, and sea surface height gradients using a generalize additive model. This and follow-on work combined acoustic surveys and in situ sampling to verify sardine presence 14,41. In previous work, generalized 

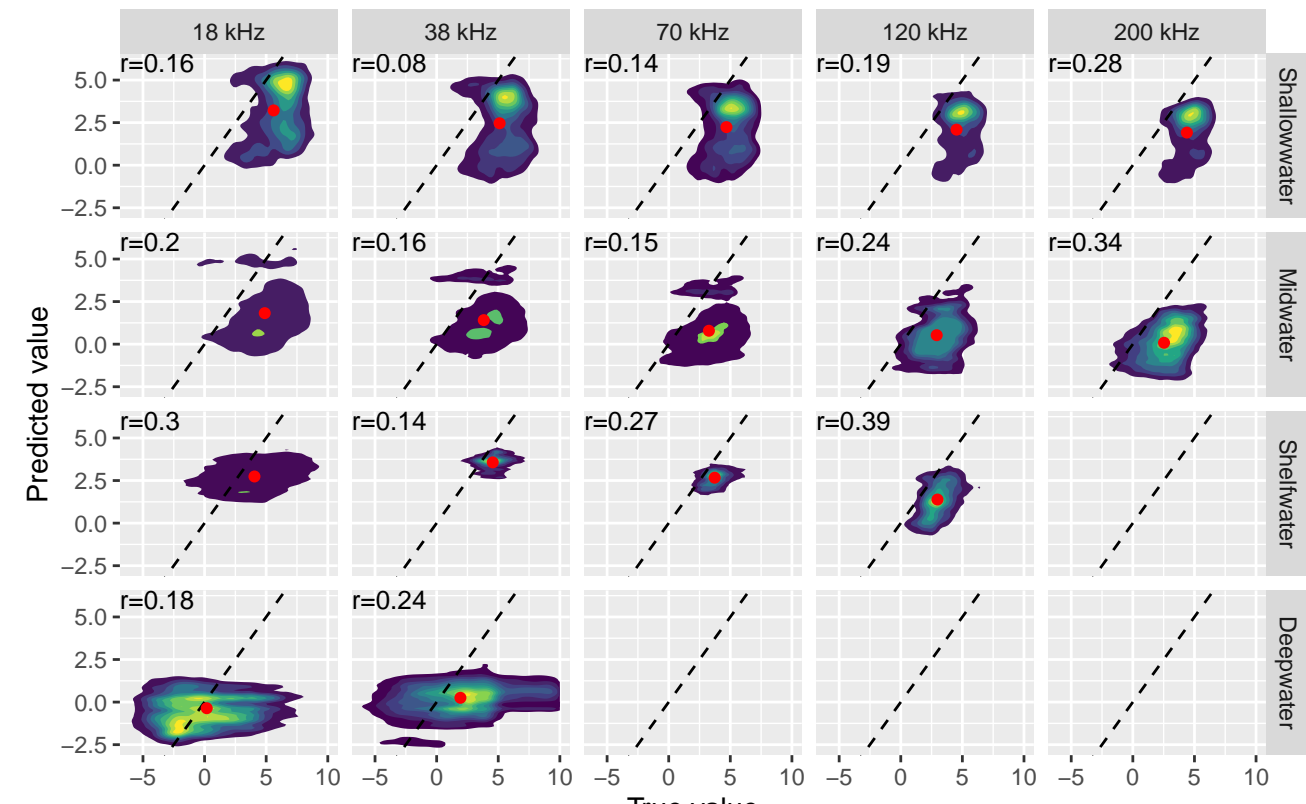

$r=0.15$
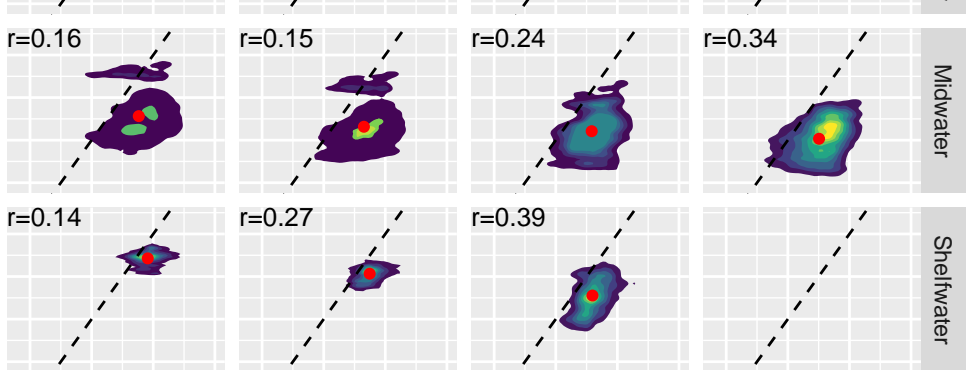

$r=0.27$
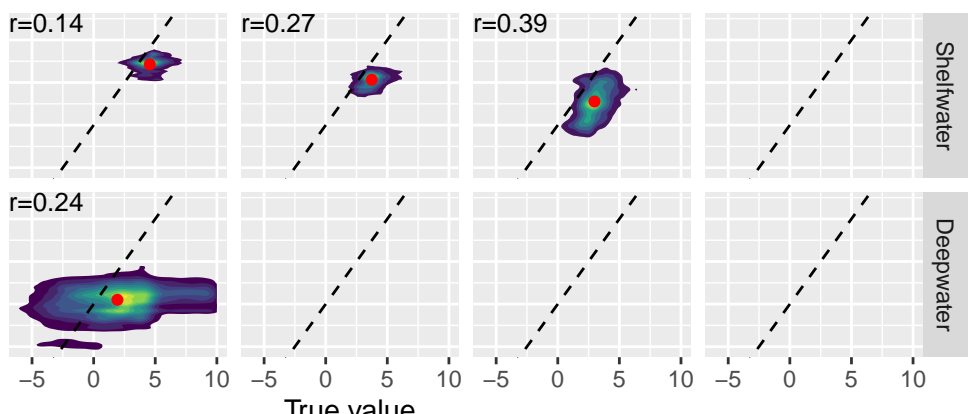

Fig 4. Observed vs. true values in the test set. Density surface plots for the relationship between predicted and true (observed) log NASC values in 584,917 observations from the withheld 2015 test set and the full model, by depth bin (panel rows) and sonar frequency in $\mathrm{kHz}$ (panel columns). Yellow represents high point density, and dark blue represents low point density. A dashed one-to-one line is shown in every panel, the mean values of true and predicted log NASC are shown as red dots. Red dots far from the dashed line indicate bias in the mean predictions. Pearson correlation coefficients are printed in the upper left of each panel.

additive models were used to model sonar data at $70 \mathrm{kHz}$ as a function of satellite-derived chlorophyll a, sea surface temperature, spatial location, depth, and distance from shore off the Hawaii Islands [42]. Latitude, longitude, chlorophyll-a, depth, distance from shore, and sea surface temperature explained roughly $35 \%$ of variance in observed NASC [42]. However, a one-to-one comparison with this project is not possible because out of sample predictive performance was not evaluated, and the satellite data were not withheld in a reduced model.

Looking forward, it seems that embedding more mechanistic or process-based structure in an approach like the one developed here might be beneficial. A hybrid approach that combines a parametric dynamical model with a more flexible machine learning model (like a neural hierarchical model) might provide one such approach 43 . A neural hierarchical model would provide a framework for specifying a flexible model for a partly known process (e.g., hake migration) and a linked model of data collection (e.g., the location of research vessels). In contrast, an unconstrained machine learning model like a random forest does not account for spatiotemporal mismatch in the trajectory of a vessel collecting sonar data, and the spatiotemporal dynamics of biological processes.

\section{Conclusion}

Taken together, these results indicate that MODIS satellite observations data may be useful for spatial interpolation of marine sonar data in some cases, but may be 
unreliable for temporal forecasting or spatial extrapolation. We suspect that the lack of performance is a consequence of a mismatch between the observable surface dynamics of sea surface temperature and chlorophyll a with the complex spatiotemporal subsurface dynamics that relate to marine food webs and population dynamics. Three-dimensional circulation models and sustained surface features (fronts) in addition to a longer time series are recommended in future analyses.

\section{Acknowledgments}

This work was supported by the University of Colorado Boulder Grand Challenge's investment in Earth Lab, and through the Innovative Research Program (IRP) of the Cooperative Institute for Research in Environmental Science (CIRES) at the University of Colorado Boulder.

\section{References}

1. Davison PC, Koslow JA, Kloser RJ. Acoustic biomass estimation of mesopelagic fish: backscattering from individuals, populations, and communities. ICES Journal of Marine Science. 2015;72(5):1413-1424.

2. Benoit-Bird KJ, Au WW. Target strength measurements of Hawaiian mesopelagic boundary community animals. The Journal of the Acoustical Society of America. 2001;110(2):812-819.

3. Trenkel VM, Berger L. A fisheries acoustic multi-frequency indicator to inform on large scale spatial patterns of aquatic pelagic ecosystems. Ecological indicators. 2013;30:72-79.

4. Towler RH, Jech JM, Horne JK. Visualizing fish movement, behavior, and acoustic backscatter. Aquatic Living Resources. 2003;16(3):277-282.

5. Kaartvedt S, Røstad A, Klevjer TA, Staby A. Use of bottom-mounted echo sounders in exploring behavior of mesopelagic fishes. Marine Ecology Progress Series. 2009;395:109-118.

6. Jech JM, Stroman F. Aggregative patterns of pre-spawning Atlantic herring on Georges Bank from 1999-2010. Aquatic Living Resources. 2012;25(1):1-14.

7. Simmonds J, MacLennan DN. Fisheries acoustics: theory and practice. John Wiley \& Sons; 2008.

8. Lee Z, Weidemann A, Kindle J, Arnone R, Carder KL. Euphotic zone depth: Its derivation and implication to ocean-color remote sensing. J Geophys Res. $2007 ; 112$.

9. Ressler PH, Brodeur RD, Peterson WT, Pierce SD, Vance PM, Røstad A, et al. The spatial distribution of euphausiid aggregations in the Northern California Current during August 2000. Deep Sea Research Part II: Topical Studies in Oceanography. 2005;52(1-2):89-108.

10. Benoit-Bird KJ, Au WW. Diel migration dynamics of an island-associated sound-scattering layer. Deep Sea Research Part I: Oceanographic Research Papers. 2004;51(5):707-719. 
11. Santora JA, Ralston S, Sydeman WJ. Spatial organization of krill and seabirds in the central California Current. ICES Journal of Marine Science. 2011;68(7):1391-1402.

12. Demer DA, Zwolinski JP, Byers KA, Cutter GR, Renfree JS, Sessions TS, et al. Prediction and confirmation of seasonal migration of Pacific sardine (Sardinops sagax) in the California Current Ecosystem. Fishery Bulletin. 2012;110(1):52-70.

13. Ressler PH, Holmes JA, Fleischer GW, Thomas RE, Cooke KC. Pacific hake, Merluccius productus, autecology: a timely review. Marine Fisheries Review. 2007;69(1):1.

14. Zwolinski JP, Emmett RL, Demer DA. Predicting habitat to optimize sampling of Pacific sardine (Sardinops sagax). ICES Journal of Marine Science. 2011;68(5):867-879.

15. NWFSC. Water Column Sonar Data Collection (SH1103, EK60), National Centers for Environmental Information, NOAA; 2011. Available from: doi:10.7289/V53J39XH

16. NWFSC. Water Column Sonar Data Collection (SH1204, EK60), National Centers for Environmental Information, NOAA; 2012. Available from: doi:10.7289/V5ZS2TF7.

17. NWFSC. Water Column Sonar Data Collection (SH1305, EK60), National Centers for Environmental Information, NOAA; 2013. Available from: doi:10.7289/V57942N7.

18. NWFSC. Water Column Sonar Data Collection (SH1507, EK60), National Centers for Environmental Information, NOAA; 2015. Available from: doi:10.7289/V5D50JZQ

19. Foote KG, Knudsen HP, Vestness G, Maclennan DN, Simmonds J. Calibration of acoustic instruments for fish density estimation: A practical guide. ICES Cooperative Research Report. 1987;144:1-69.

20. De Robertis A, Higginbottom I. A post-processing technique to estimate the signal-to-noise ratio and remove echosounder background noise. ICES Journal of Marine Science. 2007;64(6):1282-1291.

21. Ryan TE, Downie RA, Kloser RJ, Keith G. Reducing bias due to noise and attenuation in open-ocean echo integration data. ICES Journal of Marine Science. 2015;72(8):2482-2493.

22. MacLennan DN, Fernandes PG, Dalen J. A consistent approach to definitions and symbols in fisheries acoustics. ICES Journal of Marine Science. 2002;59(2):365-369.

23. Whitehead PJ. Clupeoid Fishes of the World (Suborder Clupeoidei): An annotated and illustrated catalogue of the herrings, sardines, pilchards, sprats, shads, anchovies and wolf-herrings. vol. 7. FAO; 1985.

24. De Robertis A, McKelvey DR, Ressler PH. Development and application of an empirical multifrequency method for backscatter classification. Canadian Journal of Fisheries and Aquatic Sciences. 2010;67(9):1459-1474. 
25. Korneliussen RJ, Ona E. An operational system for processing and visualizing multi-frequency acoustic data. ICES Journal of Marine Science. 2002;59(2):293-313.

26. Benoit-Bird KJ. The effects of scattering-layer composition, animal size, and numerical density on the frequency response of volume backscatter. ICES Journal of Marine Science. 2009;66(3):582-593.

27. NASA Goddard Space Flight Center OBPG Ocean Ecology Laboratory. Moderate-resolution Imaging Spectroradiometer (MODIS) Aqua and Terra ocean color data; 2014.

28. GEBCO. GEBCO 30 arc-second grid; 2014. Available from: https://www.gebco.net/data_and_products/gridded_bathymetry_data/ gebco_30_second_grid/.

29. Di Lorenzo E, Mantua N. Multi-year persistence of the 2014/15 North Pacific marine heatwave. Nature Climate Change. 2016;6(11):1042-1047.

30. Wright MN, Ziegler A. ranger: A Fast Implementation of Random Forests for High Dimensional Data in C++ and R. Journal of Statistical Software. 2017;77(1):1-17. doi:10.18637/jss.v077.i01.

31. R Core Team. R: A Language and Environment for Statistical Computing; 2019. Available from: https://www.R-project.org/.

32. Joseph M, Gadeken K, McGlinchy J, Karnauskas KB, C Wall C. Sonar data from Gadeken et al. 2020; 2020. Available from: https://figshare.com/articles/ Sonar_data_from_Gadeken_et_al_2020/12397958/1.

33. Taylor AG, Goericke R, Landry MR, Selph KE, Wick DA, Roadman MJ. Sharp gradients in phytoplankton community structure across a frontal zone in the California Current Ecosystem. Journal of Plankton Research. 2012;34(9):778-789.

34. Powell JR, Ohman MD. Covariability of zooplankton gradients with glider-detected density fronts in the Southern California Current System. Deep Sea Research Part II: Topical Studies in Oceanography. 2015;112:79-90.

35. Chen L, Li T, Wang B, Wang L. Formation mechanism for 2015/16 super El Niño. Scientific reports. 2017;7(2975).

36. Hu S, Fedorov AV. The extreme El Niño of 2015-2016: the role of westerly and easterly wind bursts, and preconditioning by the failed 2014 event. Climate Dynamics. 2019;52:7339-7357.

37. Jacox MG, Hazen EL, Zaba KD, Rudnick DL, Edwards CA, Moore AM, et al. Impacts of the 2015-2016 El Niño on the California Current System: Early assessment and comparison to past events. Geophysical Research Letters. 2016;43(13):7072-7080.

38. Zaba K, Rudnick DL, Cornuelle B, Gopalakrishnan G, Mazloff M. Volume and heat budgets in the coastal California Current System: Means, annual cycles and interannual anomalies of 2014-2016. Journal of Physical Oceanography. 2020;2020.

39. Liu M, Liu X, Liu D, Ding C, Jiang J. Multivariable integration method for estimating sea surface salinity in coastal waters from in situ data and remotely sensed data using random forest algorithm. Computers \& geosciences. 2015;75:44-56. 
40. Song W, Dolan JM, Cline D, Xiong G. Learning-based algal bloom event recognition for oceanographic decision support system using remote sensing data. Remote Sensing. 2015;7(10):13564-13585.

41. Demer DA, Zwolinski JP. Corroboration and refinement of a method for differentiating landings from two stocks of Pacific sardine (Sardinops sagax) in the California Current. ICES Journal of Marine Science. 2014;71(2):328-335.

42. Copeland AM. Influences of spatial and temporal variability of sound scattering layers on deep diving odontocete behavior $[\mathrm{PhD}]$. University of Hawaii at Manoa. 2500 Campus Rd, Honolulu, HI 96822; 2016.

43. Joseph MB. Neural hierarchical models of ecological populations. Ecology Letters. 2020;23:734-747. 